\title{
Keep the old, in with the new: The changing face of pleural effusions
}

Pleural effusion (PE) is a collection of fluid in the pleural space and is a common complication of pneumonia in children. Despite advances in prevention and management, pneumonia remains a leading cause of morbidity and mortality, accounting for $15 \%$ of deaths in children under 5 years worldwide. ${ }^{[1]}$ Developing countries are disproportionately affected as more than $95 \%$ of all global cases of clinical pneumonia occur in developing countries, particularly in sub-Saharan Africa. ${ }^{[2,3]}$ In this context, improvements in the incidence and severity of pneumonia in children have been achieved due to strategies such as reducing paediatric HIV and immunisation with conjugate vaccines. However, these successes are threatened by a lack of diagnostic infrastructure, human resources, and funding.

The introduction of the 7-valent pneumococcal conjugate vaccine (PCV7) in 2009 and PCV13 in 2011 catalysed a reduction of $89 \%$ and $57 \%$ of the respective serotype-related invasive pneumococcal disease incidence in South African children as early as $2012{ }^{[4]}$ At the same time, a meta-analysis of pragmatic studies worldwide similarly demonstrated a reduction of $31 \%$ and $17 \%$ of clinically and radiologically confirmed pneumonia and hospitalisation rates, respectively. ${ }^{[5]}$ Furthermore, and as expected, an investigation of the 8 years after the introduction of PCV at a Cape Town tertiary paediatric hospital highlighted sustained effectiveness and halving of Streptococcus pneumoniae-related PE. ${ }^{[6]}$

In this issue of the AJTCCM, Golden et al. ${ }^{[7]}$ published data from a 3 -year review of children presenting with PE in Cape Town. Of interest is that the incidence of $\mathrm{PE}$ has declined to 5.6 per 1000 children with pneumonia, and the proportion of those with $S$. pneumoniae infection has declined significantly, with S. pneumoniae accounting only for $8 \%$ of PE. ${ }^{[7]}$ Staphylococcus aureus (S. aureus) has emerged as the predominant bacterial cause of PEs, and all of these were cloxacillin sensitive. They also found that pulmonary tuberculosis (TB) accounted for almost $40 \%$ of PEs. This is a high number but considering the high incidence of TB in Cape Town ( 500/100 000), it highlights the importance of investigating and excluding $\mathrm{TB}$ as a potential cause of PE.

A fifth of children in the study had unclassified PE (no organism was identified on microscopy, culture or sensitivity). In contrast to previous culture-based studies, PCR testing afforded a higher sensitivity for detecting S. pneumoniae. However, in the study by Golden et al., ${ }^{[7]}$ molecular testing was limited due to a lack of funding. A study conducted in a developed country on the epidemiology of PE before the introduction of PCV13 found that molecular diagnostic testing significantly increased the identification of pathogens in $84 \%$ of samples from 63 paediatric patients with $\mathrm{PE}$ compared with only $35 \%$ of samples using conventional culture methods $(p<0.001)$. ${ }^{[8]}$ Secondly, dual infections involving $S$. pneumoniae in patients with cultures positive for a single pathogen were noticed. Thirdly, S. pneumoniae was the most common cause of culture-negative PE. Lastly, but perhaps most importantly, the study also highlighted the potential for bias when relying on culture alone for epidemiological studies, as $S$. aureus was most likely isolated in culture compared with $S$. pneumoniae and bacterial pathogens commonly susceptible to penicillins were identified by PCR. So, a future study using PCR and serotyping in the reported cohort in Cape Town may assist in increasing the yield and improve organism identification for better targeted treatment.

What is probably of more practical guidance for treating clinicians is the characterisation of the clinical presentation and laboratory results of participants with PE. As expected, TB should be suspected in those with chronic cough and weight loss and in children who are older at presentation. Infants with higher serum inflammatory markers at presentation and higher pleural fluid protein and adenosine deaminase (ADA) were more likely to have bacterial infection and should include S. aureus cover.

Molecular diagnostics are informing understanding of the changing epidemiology in PE in countries implementing their use. Future studies should attempt to characterise the role of PCR in epidemiological studies to improve our understanding of the spectrum of pathogens in a high TB setting.

R E M Mphahlele, MB ChB, MMedSci; R Masekela, MB ChB, PhD Department of Paediatrics and Child Health, School of Clinical Medicine, College of Health Sciences, University of KwaZulu-Natal, Durban, South Africa. masekelar@ukzn.ac.za

Afr J Thoracic Crit Care Med 2021;27(4):131. https://doi. org/10.7196/AJTCCM.2021.v27i4.182

1. World Health Organization. WHO causes of child mortality 2017. Geneva: WHO, 2017. https://www.who.int/gho/child_health/mortality/causes/en/ (accessed 13 October 2021).

2. Rudan I, Tomaskovic L, Boschi-Pinto C, et al. Global estimate of the incidence of clinical pneumonia among children under five years of age. Bull World Health Organ 2004;82:895-903.

3. Walker CL, Rudan I, Liu L, et al. Global burden of childhood pneumonia and diarrhoea. Lancet 2013;381(9875):1405-1416. https://doi.org/10.1016/S01406736(13)60222-6

4. Von Gottberg A, De Gouveia L, Tempia S, et al. Effects of vaccination on invasive pneumococcal disease in South Africa. N Engl J Med 2014;371(20):1889-1899. https://doi.org/10.1056/NEJMoa1401914

5. Alicino C, Paganino C, Orsi A, et al. The impact of 10-valent and 13-valent pneumococcal conjugate vaccines on hospitalisation for pneumonia in children: A systematic review and meta-analysis. Vaccine 2017;35(43):5776-5785. https://doi. org/10.1016/j.vaccine.2017.09.005

6. Zampoli M, Kappos A, Wolter N, et al. Aetiology and incidence of pleural empyema in South African children. Paediatr Infect Dis J 2015;34(12):1305-1310. https://doi. org/10.1097/INF.0000000000000880

7. Golden L, Chaya S, Reitchmuth K, et al. Aetiology and presentation of childhood pleural infections in the post-pneumococcal conjugate vaccine era in South Africa. Afr J Thoracic Crit Care Med 2021.;27(4):138-144. https://doi.org/10.7196/ AJTCCM.2021.v27i4.152

8. Blaschke AJ, Heyrend C, Byington CL, et al. Molecular analysis improves pathogen identification and epidemiologic study of paediatric parapneumonic empyema. Paediatr Infect Dis J 2011;30(4):289. https://doi.org/10.1097/INF.0b013e3182002d14 